\title{
UN EQUILIBRIO BAYESIANO DE NASH: COMPETENCIA A LA COURNOT BAJO INFORMACIÓN ASIMÉTRICA Y PRODUCTOS DIFERENCIADOS ${ }^{1}$
}

\author{
ALEJANDRO RAMÍREZ VIGOYA ${ }^{2}$ \\ UNIVERSIDAD MILITAR NUEVA GRANADA
}

Recibido/ Received/ Recebido: 23-05-2011 - Aceptado/ Accepted / Aprovado: 24-10-2011

\begin{abstract}
Resumen
Este artículo es un aporte teórico en dos campos de la economía: teoría de juegos y organización industrial. Se halla un equilibrio bayesiano de Nash-Cournot cuando dos empresas compiten en cantidades producidas. Una de las empresas tiene información completa sobre sus costos y los de su competidora, pero la otra empresa solo conoce los costos propios y le asigna probabilidades a los posibles costos de su competidora. Los bienes producidos por las empresas pueden ser desde completamente homogéneos hasta completamente heterogéneos. El grado de sustitución de los productos determina los efectos en el equilibrio, tanto de los costos propios como de los costos de la otra empresa en las cantidades de equilibrio. El análisis de estática comparativa del equilibrio, indica que los costos propios de cada empresa hacen que disminuyan en mayor proporción las cantidades de equilibrio cuanto mayor sea el grado de sustituibilidad de los productos. Por otro lado, los costos de la otra empresa hacen que aumente en mayor proporción las cantidades de equilibrio, a medida que los productos son más homogéneos o sustituibles.
\end{abstract}

Palabras clave: Equilibrio Bayesiano, Equilibrio de Nash-Cournot, Productos diferenciados, Organización Industrial

\section{UN EQUILIBRIO BAYESIANO DE NASH: COMPETENCIA A LA COURNOT BAJO INFORMACIÓN ASIMÉTRICA Y PRODUCTOS DIFERENCIADOS}

\begin{abstract}
This article is a theoretical contribution in two fields of economics: game theory and industrial organization. In this article Nash-Cournot Bayesian equilibrium is calculated when two companies compete on produced quantities. One of the companies has full information on its costs and its competitor, but the other company just knows its own costs and assigns probabilities to possible costs of its competitor. Produced goods by the companies can be from completely homogeneous to completely heterogeneous. Product substitution degree determines the equilibrium effects, of both own costs and other company's costs in equilibrium quantities. The comparative static analysis of balance indicates that own costs for each company make that balance quantities decrease in a greater proportion when product substitution degree is higher. On the other hand, other company's costs increase balance quantities to the extent that products are more homogenous or replaceable.

Keywords: Bayesian equilibrium, Nash-Cournot equilibrium, differentiated productos, industrial organization.

1 Este artículo es un resultado teórico de la línea de investigación sobre políticas públicas, perteneciente al grupo de investigación del Centro de Investigaciones Económicas (CIE)

2 Profesor de Macroeconomía Universidad Militar Nueva Granada. Correo electrónico: alejandro.ramirez@unimilitar.edu.co
\end{abstract} alej-ram@uniandes.edu.co 


\title{
UM EQUILÍBRIO BAYESIANO DE NASH: CONCORRÊNCIA À COURNOT SOB INFORMAÇÃO ASSIMÉTRICA E PRODUTOS DIFERENCIADOS
}

\begin{abstract}
Resumo
Este artigo é um aporte teórico em dois campos da economia: teoria de jogos e organização industrial. Apresenta-se um equilibrio bayesiano de Nash-Cournot quando duas empresas competem em quantidades produzidas. Uma das empresas tem informação completa sobre seus custos e sobre os de sua concorrente. Mas a outra empresa só conhece os custos próprios e atribui probabilidades aos possiveis custos de sua concorrente. Os bens produzidos pelas empresas podem ser desde completamente homogéneos até completamente heterogêneos. O grau de substituição dos produtos determina os efeitos no equilibrio, tanto dos custos próprios como dos custos da outra empresa nas quantidades de equilibrio. A análise de estática comparativa do equilibrio indica que os custos próprios de cada empresa fazem com que diminuam em maior proporção as quantidades de equilíbrio quanto maior for o grau de substituição dos produtos. Por outro lado, os custos da outra empresa fazem com que aumente em maior proporção as quantidades de equilîbrio à medida que os produtos forem mais homogéneos ou substituíveis.
\end{abstract}

Palavras chave: Equilíbrio Bayesiano, Equilíbrio de Nash-Cournot, Produtos diferenciados, Organização Industrial.

Ramírez, A. (2011) Un equilibrio bayesiano de Nash: competencia a la Cournot bajo información asimétrica y productos diferenciados. En: Revista de la Facultad de Ciencias Económicas de la Universidad Militar Nueva Granada. rev.fac.cienc.econ, XIX (2)

JEL: D23, D43, D83

\section{Introducción}

En la literatura de modelos oligopólicos de mercado con productos homogéneos existen modelos cuando las empresas compiten en precios y cuando las empresas compiten en cantidades producidas. En competencia en precios, Bertrand (1883) fue uno de los precursores, al encontrar un equilibrio en precios cuando un grupo de empresas compiten al producir un bien homogéneo, es decir, los bienes son sustitutos perfectos. En este modelo, los consumidores compran el bien con el precio más bajo y las empresas compiten hasta que el precio se iguala a los costos marginales, momento en el cual, el equilibrio de Bertrand se convierte en equilibrio bajo competencia perfecta.

Los modelos de oligopolio y competencia en cantidades producidas fueron tratados inicialmente por
Cournot (1838). Cournot encontró un equilibrio estable cuando dos o más empresas compiten en cantidades producidas, teniendo costos marginales iguales y productos homogéneos. El equilibrio de Cournot se presenta cuando las empresas maximizan beneficios, y las cantidades óptimas producidas se encuentran cuando la función de reacción de cada una, es la mejor respuesta a las cantidades producidas por las otras empresas.

En su trabajo doctoral, Nash (1950), encontró un equilibrio en el cuál el valor óptimo de cada jugador es la mejor respuesta a las estrategias de los otros jugadores. El equilibrio de Nash, le mereció el premio Nóbel de economía en 1994. El equilibrio de Nash se presenta en un juego en forma normal con $n$ jugadores, $\mathrm{G}=\left\{\mathrm{S}_{1}, \ldots, \mathrm{S}_{\mathrm{n}} ; \mathrm{u}_{1}, \ldots, \mathrm{u}_{\mathrm{n}}\right\}$, entonces las estrategias $\mathrm{s}_{1}{ }^{*}, \ldots, \mathrm{s}_{\mathrm{n}}$ * forman un equilibrio de Nash si, para cada jugador $\mathrm{i}, \mathrm{s}_{\mathrm{i}}{ }^{*}$ es la mejor respuesta del 
jugador i a las estrategias de los otros $\mathrm{n}-1$ jugadores, $\left(\mathrm{s}_{1}^{*}, \ldots, \mathrm{s}_{\mathrm{i}-1}{ }^{*}, \mathrm{~s}_{\mathrm{i}+1} *, \ldots, \mathrm{s}_{\mathrm{n}}^{*}\right)$. El equilibrio de Nash con dos jugadores y estrategias puras coincide con el equilibrio de Cournot cuando compiten dos empresas en cantidades producidas.

En el equilibrio de Nash-Cournot, cuando compiten dos empresas en cantidades producidas, existe información incompleta o asimétrica cuando una de las empresas no posee toda la información que la otra empresa conoce. El equilibrio resultante es conocido como equilibrio bayesiano de Nash o juegos bayesianos con información incompleta, tema que ha sido trabajado entre otros autores por Harsanyi (1967), quien analiza juegos en los cuales los jugadores (empresas) no tienen certeza sobre ciertos parámetros de la situación de un juego, como las funciones de pagos, las estrategias disponibles, etc.

Por otro lado, en teoría de la organización industrial, el tema de la diferenciación de los bienes producidos cuando dos o más empresas compiten ha sido ampliamente cubierto en modelos bajo monopolio, competencia monopolística y oligopolios; en los cuales, uno de los supuestos principales, es precisamente, que las empresas que compiten se diferencian de las otras por cierto grado de diferenciación de los bienes producidos. De acuerdo a la forma como se diferencian los productos, los modelos se pueden agrupar en tres tipos: diferenciación vertical, diferenciación horizontal y diferenciación debido a las características propias de los bienes.

En los modelos de diferenciación vertical y horizontal, los consumidores solamente tienen la opción de comprar un bien. Una de las principales características de la diferenciación vertical es la calidad del producto, que se da en los monopolios que discriminan precios; en estos modelos los consumidores eligen determinado producto por la calidad del mismo, donde el precio puede ser una señal de la calidad (Allen, 1984; Chan \& Leland, 1982; Kihlstrom \& Levhari, 1977).

Existen modelos oligopólicos de diferenciación vertical, donde la calidad y el precio determinan la de- manda de los consumidores, y la demanda óptima se encuentran cuando éstos maximizan sus utilidades (Gabszewics \& Thisse, 1979; Shaked \& Sutton, 1982).

La diferenciación horizontal se presenta cuando dos o más empresas que producen el mismo bien son elegidas por los consumidores de acuerdo a su localización espacial en determinado sitio (Hotelling, 1929; Salop, 1979). Cuando los consumidores tienen la opción de comprar muchos bienes, lo que les puede interesar son las características de una canasta de bienes producidos en diferentes sectores (Lancaster, 1966).

Dentro de la teoría existente en competencia con diferenciación de productos, están los modelos bajo competencia monopolística, uno de cuyos precursores fue Chamberlin (1933). Estos modelos se caracterizan por que las empresas enfrentan funciones de demanda bastante elásticas y cualquier variación en el precio de uno de los productos hace que su demanda aumente más que proporcionalmente. Más recientemente, Dixit \& Stiglitz (1977) y Spence (1976), plantean modelos donde la sustituibilidad de los productos no se debe a diferenciación vertical $u$ horizontal. En estos modelos existe un consumidor representativo y además no existe heterogeneidad en los gustos. El consumidor demanda una proporción pequeña de cada producto disponible en lugar de un solo bien.

El modelo que se plantea y resuelve, en el presente trabajo, es un equilibrio bayesiano de Nash-Cournot, cuando compiten dos empresas en cantidades producidas, en el cual, los productos pueden ser desde sustitutos perfectos, hasta completamente diferentes, es decir desde completamente homogéneos hasta totalmente heterogéneos. Es un equilibrio bayesiano porque existe información incompleta o asimétrica para una de las empresas. Una de las empresas conoce sus costos y los de la otra empresa, pero la otra empresa le asigna probabilidades a los costos de la primera, ya que no tiene certeza de con cuales costos funciona. 
Es un equilibrio de Nash debido a que las cantidades de equilibrio encontradas, para cada empresa, son la mejor respuesta a las cantidades producidas por la otra empresa. Es decir, las cantidades producidas de equilibrio de cada empresa son la mejor opción dentro de múltiples opciones de producción que tienen las empresas.

Es un equilibrio de Cournot por que el equilibrio encontrado es la intercepción de las respectivas funciones de reacción de las dos empresas. Las funciones de reacción de cada empresa son la mejor respuesta de las cantidades producidas por una de las empresas frente las cantidades producidas por la otra.

En este trabajo la diferenciación del producto se presenta en el modelo de duopolio, debido a que las empresas compiten en cantidades producidas, pero los bienes podrían ser desde sustitutos perfectos hasta completamente diferentes. La diferenciación del producto no es ni vertical ni horizontal, debido a que los productos son de similar calidad y no importa la ubicación espacial de las empresas. En el modelo planteado en este trabajo se considera que solo existen dos empresas cuyos productos son de calidad similar, pero físicamente pueden ser desde sustitutos perfectos hasta completamente diferenciados.

La primera parte de este trabajo es esta introducción. En la segunda parte se plantea y encuentra un equilibrio bayesiano estático de duopolio de NashCournot para empresas que compiten en cantidades producidas de bienes que pueden ser desde sustitutos perfectos hasta completamente diferenciados. Una de las empresas tiene información incompleta, que se presenta cuando una de las empresas no posee toda la información sobre los costos de la otra empresa y le asigna probabilidades a los posibles costos (altos o bajos) de la otra empresa.
También se encuentran otros equilibrios que se pueden deducir a partir del inicial, bajo diferentes supuestos iniciales como son: equilibrio bayesiano de Nash-Cournot con información incompleta y productos no homogéneos, equilibrio bayesiano de Nash-Cournot con información incompleta y productos homogéneos o heterogéneos, equilibrio de Nash-Cournot con información completa, productos no homogéneos y costos diferentes, equilibrio de Nash-Cournot con productos no homogéneos y costos iguales, equilibrio de Nash-Cournot con productos homogéneos o heterogéneos y costos iguales, equilibrio de Nash-Cournot con productos homogéneos o heterogéneos y costos diferentes, entre otros.

En la tercera parte se presentarán las conclusiones del modelo teórico e implicaciones del mismo.

\section{Modelo}

\subsection{Funciones de Demanda}

La empresa dos enfrenta una función lineal e inversa de demanda ${ }^{3}$, de la forma:

$$
p_{2}=a-s q_{1}-q_{2}
$$

Donde $\mathrm{p}_{2}$ es el precio al cual vende la empresa dos, que depende de la cantidad que produzca ella misma, $\mathrm{q}_{2}$, y de las cantidades que produzca la empresa uno, $\mathrm{q}_{1}$ Las cantidades de la empresa uno están multiplicadas por un parámetro $\mathrm{s}^{4}$, que mide el grado de homogeneidad de los productos que venden las dos empresas. Si s es igual a uno, los productos son completamente homogéneos, si s es igual a cero los productos son heterogéneos y las funciones de demanda se convierten en monopólicas. El parámetro s está entre cero y uno, entre más cercano este a uno,

La función inversa de demanda es la que utiliza Cournot para modelos de oligopolio: $\mathrm{P}=\mathrm{a}-\mathrm{Q}$, donde $\mathrm{Q}=\mathrm{sq}_{1}+\mathrm{q}_{2}$, dado que es un duopolio. El parámetro s mide el grado de diferenciación de los productos.

4 El grado de diferenciación de los dos productos lo da el valor del parámetro s, si s es igual a uno el modelo se convierte en un equilibrio bayesiano de Nash-Cournot con productos homogéneos, si s es igual a cero los bienes son completamente diferentes y las empresas funcionan como monopolios. Pero s podría tomar un valor entre cero y uno, si es cercano a uno los productos son altamente sustituibles, si es cercano a cero los productos no son tan sustituibles. 
los productos son más parecidos, entre más cercano a cero, los productos son más diferenciados.

La empresa dos puede fabricar con dos tipos de costos por unidad producida: costos altos $\mathrm{C}_{2}\left(\mathrm{q}_{2}\right)=$ Caq ${ }_{2}$ o costos bajos $\mathrm{C}_{2}\left(\mathrm{q}_{2}\right)=\mathrm{Cbq}_{2}^{5}$. La asimetría en la información o, también denominada información incompleta, consiste en que la empresa dos puede trabajar con unos u otros (costos altos o bajos) y la empresa uno al no tener certidumbre de con que costos trabaja la empresa dos, entonces le asigna probabilidades de que trabaje con unos o con otros ${ }^{6}$.

La empresa uno tiene dos funciones inversas y lineales de demanda?:

$$
\begin{aligned}
& p_{1}=a-q_{1}-s q_{2}(C a) \\
& p_{1}=a-q_{1}-s q_{2}(C b)
\end{aligned}
$$

Donde $\mathrm{q}_{2}(\mathrm{Ca})$ son las cantidades que produce la empresa dos, cuando trabaja con costos altos y $\mathrm{q}_{2}(\mathrm{Cb})$ son las cantidades que produce la empresa dos, cuando trabaja con costos bajos ${ }^{8}$.

La empresa uno, asigna una probabilidad $\theta$ a que la empresa dos trabaje con costos altos (Ca) y (1- $\theta$ ) a que la empresa dos trabaje con costos bajos $(\mathrm{Cb})^{9}$. El parámetro s en estas funciones de demanda tiene el mismo papel que el que tiene en la función de demanda de la empresa dos.

\subsection{Funciones de Beneficio}

La empresa dos maximiza beneficios ${ }^{10}$ dependiendo de los costos que tenga; pero solo ella conoce los costos que tiene. Debido a esto tiene dos funciones de beneficio, una cuando trabaja con costos altos (Ca) y otra cuando trabaja con costos bajos (Cb):

$$
\begin{aligned}
& \Pi_{2}(C a)=\left[\left(a-s q_{1}-q_{2}-C a\right) q_{2}\right] \\
& \Pi_{2}(C b)=\left[\left(a-s q_{1}-q_{2}-C b\right) q_{2}\right] \\
& 0 \leq s \leq 1
\end{aligned}
$$

La empresa uno maximiza beneficios, que dependen de los costos con los que trabaje la empresa dos y de sus probabilidades; por lo tanto la función de beneficios de la empresa uno es la siguiente:

$$
\begin{aligned}
& \Pi_{1}=\theta\left[\left(a-q_{1}-s q_{2}(C a)\right)-c\right] q_{1}+(1-\theta)\left[\left(a-q_{1}-s q_{2}(C b)\right)-c\right] q_{1} \\
& 0 \leq \theta \leq 1
\end{aligned}
$$

Donde c son los costos por unidad producida de la empresa uno.

Si existiera información completa; es decir, si la empresa uno supiera con que costos $\left(\mathrm{c}_{2}\right)$ trabaja la empresa dos, por ejemplo costos altos, entonces $\mathrm{C}_{2}=$ $\mathrm{Ca}, \theta$ sería igual a uno y 1- $\theta$ igual a cero. En este caso los beneficios de la empresa uno serían sencillamente: $\Pi_{1}=\left[\left(a-q_{1}-s q_{2}-C_{1}\right) q_{1}\right]$ y los de la empresa dos: $\Pi_{2}=\left[\left(a-s q_{1}-q_{2}-C_{2}\right) q_{2}\right]$ si los costos de las empresas uno son $\mathrm{C}_{1}$ y los de la empresa dos son $\mathrm{C}_{2}$.

\subsection{Funciones de Reacción}

Derivando (4) y (5) con respecto a $\mathrm{q}_{2}$ y despejando $\mathrm{q}_{2}$ resultan las funciones de reacción (7) y (8) para la empresa dos. Derivando (6) con respecto a $\mathrm{q}_{1} \mathrm{y}$ despejando $\mathrm{q}_{1}$ se obtiene la función de reacción (9) de la empresa uno.

$$
\begin{aligned}
& q_{2}(\mathrm{Ca})=\left(a-s q_{1}-\mathrm{Ca}\right) / 2 \\
& q_{2}(\mathrm{Cb})=\left(a-s q_{1}-C b\right) / 2 \\
& q_{1}=\left[\theta\left(a-s q_{2}(\mathrm{Ca})-c\right)+(1-\theta)\left(a-s q_{2}(C b)-c\right)\right] / 2
\end{aligned}
$$

\footnotetext{
5 Para facilitar la solución del modelo, los costos de las empresas son variables y lineales con respecto a las cantidades producidas. Aunque podría incluir costos fijos y ser no lineal, pero esto dificultaría la resolución del modelo.

Realmente las probabilidades se introducen en la función de beneficios de la empresa uno.

7 Son funciones inversas, dado que la variable dependiente son los precios y no las cantidades.

$8 \quad \mathrm{q}_{2}(\mathrm{Ca})$ en este modelo no significa que las cantidades de la empresa dos dependen de los costos, lo que significa es que son las cantidades producidas por la empresa dos cuando los costos son altos. Igual aclaración es para $\mathrm{q}_{2}(\mathrm{Cb})$.

9 No existe información en la empresa uno para asignar estas probabilidades, la asignación es aleatoria.

10 Las funciones de beneficio son $\pi=$ Ingreso total - Costo total $=\mathrm{P}^{*} \mathrm{Q}-\mathrm{C}^{*} \mathrm{Q}=(\mathrm{P}-\mathrm{C}) * \mathrm{Q}$, donde $\mathrm{P}$ es la función inversa de demanda y $\mathrm{C}$ los costos unitarios con los que trabaja la respectiva empresa.
} 
Donde $\mathrm{q}_{2}(\mathrm{Ca})$ y $\mathrm{q}_{2}(\mathrm{Cb})$ es la mejor respuesta en cantidades producidas de la empresa dos, con costos altos y bajos, a las cantidades producidas por la empresa uno, $\mathrm{q}_{1}$. Y, por otro lado, $\mathrm{q}_{1}$ es la mejor respuesta en cantidades producidas de la empresa uno, a las cantidades $\mathrm{q}_{2}(\mathrm{Ca})$ y $\mathrm{q}_{2}(\mathrm{Cb})$ producidas por la empresa dos. Debido a esto el equilibrio encontrado es un equilibrio bayesiano de NashCournot entre dos empresas cuyos productos son no homogéneos.

Hay dos funciones de reacción para la empresa dos, debido a que puede trabajar con costos altos o bajos.

\subsection{Equilibrio Bayesiano de Nash-Cournot para productos no homogéneos}

Reemplazando (7) y (8) en (9), y luego de despejar $\mathrm{q}_{1}$ se halla $\mathrm{q}_{1}$ *; luego $\mathrm{q}_{1}$ * se reemplaza en (7) y (8) y se hallan $\mathrm{q}_{2}(\mathrm{Ca}) *$ y $\mathrm{q}_{2}(\mathrm{Cb})^{*}$.

El equilibrio resultante de Nash-Cournot esta representado en las funciones (10), (11) y (12) $)^{11}$ :

$$
\begin{aligned}
& q 1^{*}=a /(2+s)-[1 / \alpha(2+s)] c+[(1-\alpha) \theta / \alpha(2+s)] C a+[(1-\alpha)(1-\theta) / \alpha(2+s)] C b \quad \text { (10) } \\
& q\left(C d^{*}=a /(2+s)+[s / 2 \alpha(2+s)] c-[(2 \alpha+s) / 2 \alpha(2+s)] C a+[(1-\alpha)(1-\theta) s / 2 \alpha(2+s)](C a-C b)(11)\right. \\
& q 2(C b) *=a /(2+s)+[s / 2 \alpha(2+s)] c-[(2 \alpha+s) / 2 \alpha(2+s)] c b+[(1-\alpha) e s / 2 \alpha(2+s))((c b-C a) \\
& \begin{array}{cl}
\text { Donde } e^{12}: \quad & \alpha=1-s / 2 \\
& (1-\alpha)=s / 2
\end{array}
\end{aligned}
$$

Si $\mathrm{s}=1$, entonces $\alpha=1 / 2$ y $(1-\alpha)=1 / 2$.

Si $s=0$, entonces $\alpha=1,(1-\alpha)=0$

\subsection{Análisis de los efectos de la diferenciación del producto en las cantidades de equilibrio}

El equilibrio se puede analizar para una gama amplia de relación entre los productos de las empresas uno y dos, desde sustitutos perfectos, en este caso $\mathrm{s}$ $=1$, hasta productos que no tienen ningún grado de sustituibilidad, $\mathrm{s}=0$.

Lo más importante del equilibrio encontrado es analizar los efectos del grado de diferenciación del producto en las cantidades de equilibrio de las funciones 10,11 y 12 . En otras palabras, se quiere analizar los efectos de los costos tanto propios como cruzados de las empresas en sus cantidades de equilibrio según el grado de homogeneidad de los productos de las empresas uno y dos. Para hacer este análisis es mejor simplificar las funciones 10, 11 y 12 .

\subsubsection{Estática comparativa para la empresa 1}

Las función 10 se puede simplificar haciendo los siguientes reemplazos ${ }^{13}$ :

$$
\begin{aligned}
& \eta o=a /(2+s) \\
& \eta_{1}=1 / \alpha(2+s) \\
& \eta_{2}=(1-\alpha) \theta / \alpha(2+s) \\
& \eta_{3}=(1-\alpha)(1-\theta) / \alpha(2+s)
\end{aligned}
$$

La simplificación de la función 10 es:

$$
q_{1}^{*}=\eta o-\eta_{1} * c+\eta_{2} * C a+\eta_{3} * C b
$$

Las derivadas de las cantidades de equilibrio de la empresa uno con respecto a c, Ca y $\mathrm{Cb}$ y, las derivadas de $\eta_{1}, \eta_{2}$ y $\eta_{3}{ }^{14}$ con respecto a s, para valores de $s$ mayores que cero y menores que 1 son:

$$
\begin{array}{cc}
\partial q_{1} * / \partial c=-\eta_{1} & \partial \eta_{1} / \partial s>0 \\
\partial q_{1} * / \partial C a=\eta_{2} & \partial \eta_{2} / \partial s>0 \\
\partial q_{1} * / \partial C b=\eta_{3} & \partial \eta_{3} / \partial s>0
\end{array}
$$

Para $0 \leq s \leq 1$

\footnotetext{
En el Anexo matemático se presenta un resumen de la matemática para llegar a las ecuaciones 10,11 y 12

Se introducen estos cambios para simplificar los valores de equilibrio, pero esto no altera los análisis de estática comparativa.

La simplificación es para facilitar la comprensión del análisis de los efectos de estática comparativa

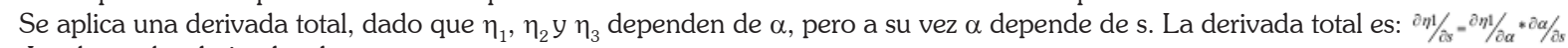
.Igual para las derivadas de $\eta_{2} y \eta_{3}$.
} 
A partir de 10.1, 10.2, 10.3 y teniendo en cuenta 10 ', se puede deducir los siguientes efectos de estática comparativa:

De 10.1 y 10 ' se puede decir que en el equilibrio encontrado de duopolio, entre más parecidos sean los productos, entonces el aumento de los costos propios de la empresa uno, hace disminuir en mayor proporción las cantidades producidas de equilibrio que si los productos son menos homogéneos.

De 10.2 y 10' se deduce que en un duopolio como el que describe este modelo, entre más parecidos sean los productos, entonces el aumento de los costos de la empresa dos (altos), hace aumentar en mayor proporción las cantidades producidas de equilibrio, de la empresa uno.

De 10.3 y 10' se puede concluir que en éste equilibrio de duopolio, entre más sustituibles sean los productos, entonces el aumento de los costos de la empresa dos (bajos), hace aumentar en mayor proporción las cantidades producidas de equilibrio, de la empresa uno.

Por otro lado, los máximos valores de $\eta_{1}, \eta_{2}$ y $\eta_{3}$ se presentan cuando s es igual a uno. Es decir, cuando los productos son sustitutos perfectos o completamente homogéneos. En este caso, se presentan los máximos efectos de los costos propios y de la otra empresa en las cantidades de equilibrio de la empresa uno. Este es un equilibrio bayesiano de NashCournot con productos homogéneos.

Cuando s es igual a cero, los productos son totalmente diferentes y no hay efectos de los costos de la empresa dos sobre las cantidades producidas por la empresa uno. En este caso la empresa uno es un monopolio.

\subsubsection{Estática comparativa para la empresa 2}

De las ecuaciones 11 y 12 se deducen los mismos efectos, por lo tanto solo se analizarán los del valor de equilibrio de la ecuación 11, que por simetría son los mismos de la ecuación 12, pero con costos bajos.

En la función 11, se pueden hacer los siguientes reemplazos ${ }^{15}$ :

$$
\begin{aligned}
& \beta o=a /(2+s) \\
& \beta_{1}=s / 2 \alpha(2+s) \\
& \beta_{2}=(2 \alpha+s) / 2 \alpha(2+s) \\
& \beta_{3}=(1-\alpha)(1-\theta) s / 2 \alpha(2+s)
\end{aligned}
$$

La simplificación de la ecuación 11 es:

$$
q_{2}\left(C d^{*}\right)=\beta o+\beta_{1} * c-\beta_{2} * C a+\beta_{3} *(C a-C b)
$$

Las derivadas de las cantidades de equilibrio de la empresa dos, con costos altos, con respecto a c y $\mathrm{Ca}$ y, las derivadas de $\beta_{1}$ y $\beta_{2}$ con respecto al parámetro $\mathrm{s}^{16}$, para valores de $\mathrm{s}$ mayores de cero y menores que 1 son:

$$
\begin{array}{ll}
\partial q_{2} * / \partial c=\beta_{1} & \partial \beta_{1} / \partial s>0 \\
\partial q_{2} * / \partial C a=-\beta_{2} & \partial \beta_{2} / \partial s>0
\end{array}
$$

Para $0 \leq s \leq 1$

A partir de 11.1 y 11.2 y teniendo en cuenta 11', se pueden deducir los siguientes efectos de estática comparativa:

De 11.1 y $11^{\prime}$ se puede analizar que en el equilibrio de duopolio encontrado, entre más parecidos sean los productos, entonces el aumento de los costos de la empresa uno, hace aumentar en mayor proporción las cantidades producidas de equilibrio, de la empresa dos.

De 11.2 y $11^{\prime}$ se deduce que en éste duopolio, entre más homogéneos sean los productos, entonces el aumento de los costos de la empresa dos (altos), hace aumentar en mayor proporción las cantidades producidas de equilibrio, de la misma empresa.

15 La simplificación se hace para facilitar el análisis de estática comparativa

16 Se aplica una derivada total, dado que $\beta_{1}$ y $\beta_{2}$ dependen de $\alpha$, pero a su vez $\alpha$ depende de s. La derivada total es: ${ }^{\partial \beta 1} / \partial s={ }^{\partial \beta 1} / \partial \alpha{ }^{*} \alpha \alpha / \partial s$ Igual para la derivada de $\beta_{2}$. 
Los máximos valores de $\beta_{1}$ y $\beta_{2}$ se presentan cuando $s$ es igual a uno. Es decir, cuando los productos son sustitutos perfectos o completamente homogéneos. En éste caso, se presentan los máximos efectos de los costos propios y de la otra empresa en las cantidades de equilibrio de la empresa dos. Éste es un equilibrio bayesiano de Nash-Cournot con productos homogéneos. Cuando s es igual a cero, los productos son totalmente diferentes y no hay efectos de los costos de la empresa uno sobre las cantidades producidas por la empresa dos. En este caso la empresa dos es un monopolio.

\section{Otros equilibrios bajo diferentes supuestos}

El equilibrio o juego estático de Nash-Cournot representado por las ecuaciones 10, 11 y 12 se puede analizar bajo diferentes supuestos:

i. Con información incompleta y productos homogéneos

ii. Con información completa y productos heterogéneos

iii. Con información completa sobre los costos y productos homogéneos

iv. Con información completa sobre los costos, productos heterogéneos y costos iguales

$v$. Con información completa sobre los costos, productos homogéneos y costos iguales

vi. Con productos totalmente heterogéneos.

\subsection{Equilibrio como un juego estático con información incompleta y productos homogéneos}

Cuando las empresas compiten en cantidades producidas y deciden simultáneamente. La información es incompleta, es decir, la empresa dos conoce sus propios costos y los de la empresa uno, pero la empresa uno le asigna probabilidades a los dos posibles costos de la empresa dos. Además, los productos son exactamente iguales, es decir, homogéneos. Para es- tos supuestos, en las funciones 10,11 y 12 se reemplaza $s=1, \alpha=1 / 2 .,(1-\alpha)=1 / 2$

El equilibrio resultante es:

$$
\begin{aligned}
& q_{1}=[a-2 c+\theta C a+(1-\theta) C b] / 3 \\
& q_{2}(C a)=(a-2 C a+c) / 3+[(1-\theta) / 6] *(C a+C b) \\
& q_{2}(C b)=(a-2 C b+c) / 3+(\theta / 6) *(C b-C a)
\end{aligned}
$$

Este equilibrio se toma como ejemplo en algunos libros de texto sobre teoría de juegos y organización industrial ${ }^{17}$.

\subsection{Equilibrio como un juego estático con información completa y productos heterogéneos}

Si las empresas compiten en cantidades y hay información completa; es decir, si la empresa dos tiene unos costos únicos, que son conocidos por ella y por la otra empresa, al igual que la empresa uno conoce los costos propios y los de la competencia. Los costos de la empresa uno son $\mathrm{C}_{1}$ y los de la empresa dos son $\mathrm{C}_{2}$. Además, en este caso el modelo se puede resolver para cualquier nivel de sustitución entre los productos de las empresas que compiten (homogeneidad perfecta hasta heterogeneidad perfecta), es decir s puede tomar cualquier valor entre 0 y 1 , entonces:

Para estos supuestos en las funciones 10, 11 y 12 se reemplaza: si $\theta=1$ entonces $C_{2}=C a$, ó si $(1-\theta)=1$ entonces $\mathrm{C}_{2}=\mathrm{Cb}$, depende de con cuáles costos conocidos trabaje la empresa dos. El parámetro s toma cualquier valor entre cero y uno.

El equilibrio resultante es:

$q_{1}=a /(2+s)+[(1-\alpha) / \alpha(2+s)] * C_{2}-[1 / \alpha(2+s)] * C_{1}$

$q_{2}=a /(2+s)+[s / 2 \alpha(2+s)] * C_{1}-[(2 \alpha+s) / 2 \alpha(2+s)] * C_{2}$

En este equilibrio los efectos del grado de homogeneidad de los productos sobre las cantidades de equilibrio, son los mismos de los del numeral 2.5.

17 Ver Gibbons (1992). 
El análisis de estática comparativa tanto para la empresa uno como para la dos, son los mismos de los numerales 2.5 .1 y 2.5.2.

\subsection{Equilibrio como un juego estático con información completa y productos homogéneos}

Este equilibrio resulta al reemplazar $\mathrm{s}=1, \alpha=1 / 2$., $(1-\alpha)=1 / 2$ en las ecuaciones 20 y 21 .

$$
\begin{array}{ll}
q_{1}=\left(a-2 C_{1}+C_{2}\right) / 3 & (22) \\
q_{2}=\left(a-2 C_{2}+C_{1}\right) / 3 & (23)
\end{array}
$$

El equilibrio resultante en 22 y 23 es conocido en la literatura. Es el clásico equilibrio de Cournot con costos diferentes y productos homogéneos que aparece regularmente en textos de teoría de juegos, de microeconomía y de organización industrial ${ }^{18}$.

\subsection{Equilibrio como un juego estático con información completa, productos heterogéneos $\mathrm{y}$ costos iguales}

Este equilibrio resulta al reemplazar en 20 y $21, C_{1}=$ $\mathrm{C}_{2}=\mathrm{c}$. El equilibrio resultante es:

$$
\begin{aligned}
& q_{1}=(a-c) /(2+s) \\
& q_{2}=(a-c) /(2+s)
\end{aligned}
$$

Haciendo un análisis de estática comparativa en 24 y 25 , los resultados son similares a los encontrados en la sección 2.5, a mayor grado de homogeneidad en los productos de las empresas uno y dos, si aumentan los costos entonces las cantidades de equilibrio disminuye en mayor proporción que si los productos son menos homogéneos.

Este equilibrio es conocido en la literatura y se utiliza en algunos libros de texto, especialmente de microeconomía (Tirole, 2001: Hirshleiter, 2000).

\subsection{Equilibrio como un juego estático con información completa, productos homogéneos y costos iguales}

Para encontrar este equilibrio se reemplaza en 24 y 25 , el parámetro s por 1 . Lo que da como resultado el siguiente equilibrio:

$$
\begin{aligned}
& q_{1}=(a-c) / 3 \\
& q_{2}=(a-c) / 3
\end{aligned}
$$

El equilibrio representado en 26 y 27 es el más común y sencillo de Nash-Cournot ${ }^{20}$

\subsection{Equilibrio como un juego estático para productos completamente heterogéneos.}

Este es el conocido equilibrio de monopolio y resulta al reemplazar en cualquier equilibrio con productos heterogéneos, el valor del parámetro s por cero.

$$
\begin{aligned}
& q_{1}=\left(a-C_{1}\right) / 2(28) \\
& q_{2}=\left(a-C_{2}\right) / 2(29)
\end{aligned}
$$

Lo que significa este equilibrio es que si dos empresas producen bienes totalmente diferenciados, entonces cada una trabaja como un monopolio para su producto ${ }^{21}$.

\section{Conclusiones}

Este trabajo hace un aporte teórico en dos campos importantes de la economía: en teoría de juegos y en organización industrial. El aporte teórico es encontrar un equilibrio bayesiano de Nash-Cournot para productos heterogéneos. En la literatura tanto de teoría de juegos como de organización industrial existían equilibrios de Nash-Cournot con productos homogéneos.

20 Este equilibrio se puede encontrar en cualquier libro de texto de microeconomía intermedia.

21 Es un equilibrio conocido en la literatura que se puede encontrar en muchos libros de texto. 
En el presente trabajo se hace un importante aporte al analizar los efectos de los costos propios y de la empresa competidora, cuando las empresas compiten en cantidades de productos con algún grado de homogeneidad y, además, existe información incompleta.

En un mercado donde existen dos empresas que compiten en cantidades producidas, es factible que una de las empresas tenga mayor información sobre los costos que la otra, por ejemplo, en el caso en el que existe una sola empresa y entra otra a competirle. En estos mercados generalmente los productos no son sustitutos perfectos, tienen cierto grado de diferenciación, por ejemplo en el caso de empresas de servicios.

En este trabajo se encuentra un equilibrio bayesiano de Nash-Cournot para dos empresas que compiten en productos donde el grado de homogeneidad puede variar desde sustitutos perfectos hasta completamente heterogéneos. Es un modelo más realista que los tradicionales donde hay información perfecta y los productos son sustitutos perfectos u homogéneos.

Los resultados de estática comparativa del equilibrio encontrado, muestran la relación que existe entre el grado de sustituibilidad de los bienes y los efectos que éste tiene en la relación entre los costos propios y los costos de la otra empresa, con respecto a las cantidades de equilibrio.

Del análisis de estática comparativa se puede decir que en el equilibrio de duopolio encontrado, entre más parecidos sean los productos, entonces el aumento de los costos de una de las empresas, hace aumentar en mayor proporción las cantidades producidas de equilibrio, de la otra empresa, más que si los productos fueran menos homogéneos. Esto se cumple aún si existe información asimétrica, de una de las empresas, sobre los costos de la otra.

También del equilibrio se deduce que en éste duopolio, entre más homogéneos sean los productos, entonces el aumento de los costos de cualquiera de las empresas, hace aumentar en mayor proporción las cantidades producidas de equilibrio, de la misma empresa, que si los productos son menos homogéneos.

El análisis de estática comparativa anterior es similar, aunque en el modelo exista información completa, $e$ inclusive cuando los costos por unidad producida sean iguales para las dos empresas.

En el equilibrio encontrado, los máximos efectos de los costos propios y de la otra empresa en las cantidades de equilibrio de cualquiera de las empresas se presentan cuando s es igual a uno. Si esto se cumple el equilibrio es bayesiano de Nash-Cournot con información incompleta y con productos homogéneos.

Cuando $s$ es igual a cero, los productos son totalmente diferentes y no hay efectos de los costos de una de las empresas sobre las cantidades producidas por la otra empresa. En éste caso las empresas son completamente independientes una de la otra, es decir, son monopolios.

\section{Referencias}

Allen, F. (1984). Reputation and Product Quality. En Rand Journal of Economics, 15: 311-327.

Bertrand, J. (1883). Théorie Mathématique de la Richesse Sociale. En Journal des Savants: 499-508.

Cournot, A. (1838). Recherches sur les Principes Mathématiques de la Théorie des Richesses. English edition (ed. N. Bacon): Researches into the Mathematical Principles of the Theory of Wealth (New York: Macmillan, 1897).

Chamberlin, E. (1933). The Theory of Monopolistic Competition. Cambridge, Mass.: Harvard University Press.

Chan, Y. \& H. Leland. (1982). Prices and Qualities in Markets with Costly Information. En Review of Economic Studies 49: 499-516.

Dixit, A., \& J. Stiglitz (1977). Monopolistic Competition and Optimum Product Diversity. En American Economic Review 67: 297-308.

Gabszewics, J. \& J.-F. Thisse, (1979). Price Competition, Quality and Income Disparities. En Journal of Economic Theory, 20: 340-359.

Gibbons, R. (1992). Un primer curso de teoría de juegos. Barcelona: Antoni Bosch Editor.

Harsanyi, J. (1967). Games with Incomplete Information Played by Bayesian Players Parts I, II and III. En Management Science, 14:159-82,320-32,486-502.

Hirshleifer, J. (2000). Microeconomía: teoría de precios y sus aplicaciones. México: Editorial Prentice-Hall.

Hotelling, H. (1929). Stability in Competition. En Economic Journal, 39: $41-57$

Kihlmstrom, R., \& D. Levhari. (1977). Quality, Regulation, Efficiency. En Kyklos, 30:114-234. 
Lancaster, K. (1966). A New Approach to Consumer Theory. En Journal of Political Economy, 74: 132-137.

Nash, J. (1950). Equilibrium Points in n-Person Games. En Proceedings of the National Academy of Sciences, 36: 85-98.

Salop, S. (1979). Monopolistic Competition with Outside Goods. En Bell Journal of Economics, 10: 141-156.
Shaked, A. \& J. Sutton. (1982). Relaxing Price Competition through Product Differentiation. En Review of Economics Studies, 49: 3-13.

Spence, M. (1976). Product Selection, Fixed Cost and Monopolistic Competition. En Review of Economic Studies, 43: 217-235. Tirole, J. (2001). The Theory of industrial Organization. Cambridge: The MIT Press.

\section{Anexo 1}

Al remplazar (7) y (8) en (9) y algunas manipulaciones matemáticas, da como resultado:

$$
q 1=\frac{a}{2}-\frac{c}{2}-\frac{a s}{4}+\frac{s^{2} q 1}{4}+\frac{\theta s C a}{4}+\frac{(1-\theta) s C b}{4}
$$

Despejando q1 y algunas manipulaciones matemáticas, se obtiene:

$$
q 1\left(1-\frac{s^{2}}{4}\right)=\frac{a}{2}\left(1-\frac{s}{2}\right)-\frac{c}{2}+\frac{\theta s C a}{4}+\frac{(1-\theta) s C b}{4}
$$

Haciendo el reemplazo:

$$
\begin{aligned}
& \alpha=1-\frac{s}{2} \\
& (1-\alpha)=\frac{s}{2} \\
& \left(1-\frac{s^{2}}{4}\right)=\left(1-\frac{s}{2}\right)\left(1+\frac{s}{2}\right)
\end{aligned}
$$

Da como resultado, la ecuación (10):

$$
q 1^{*}=a /(2+s)-[1 / \alpha(2+s)] c+[(1-\alpha) \theta / \alpha(2+s)] C a+[(1-\alpha)(1-\theta) / \alpha(2+s)] C b \text { (10) }
$$

Reemplazando (10) en (7) y algunas manipulaciones matemáticas, da como resultado:

$$
q 2(C a)=\frac{a}{2+s}+\frac{c s}{2 \alpha(2+s)}-\frac{C a}{2}\left(\frac{2 \alpha+\alpha s+(1-\alpha) \theta s}{\alpha(2+s)}\right)-\frac{(1-\alpha)(1-\theta) s C b}{2 \alpha(2+s)}
$$

Para llegar a la ecuación (11) hay que hacer un truco matemático en la anterior ecuación (se le suma y resta el término $(1-\alpha) s)$, en el numerador del paréntesis del tercer miembro del lado derecho, de tal manera que queda:

$$
q 2(C a)=\frac{a}{2+s}+\frac{c s}{2 \alpha(2+s)}-\frac{C a}{2}\left(\frac{2 \alpha+\alpha s+(1-\alpha) \theta s+(1-\alpha) s-(1-\alpha) s}{\alpha(2+s)}\right)-\frac{(1-\alpha)(1-\theta) s C b}{2 \alpha(2+s)}
$$

Manipulando matemáticamente la anterior ecuación resulta la ecuación (11)

$$
q \alpha\left(C d^{*}=a /(2+s)+[s / 2 \alpha(2+s)] c-[(2 \alpha+s) / 2 \alpha(2+s)] C a+[(1-\alpha)(1-\theta) s / 2 \alpha(2+s))(C a-C b)\right.
$$


Por otro lado reemplazando la ecuación (10) en la (8) y algunas manipulaciones matemáticas, se llega a:

$$
q 2(C b)=\frac{a}{2}-\frac{C b}{2}-\frac{a s}{2(2+s)}+\frac{c s}{2 \alpha(2+s)}-\frac{(1-\alpha) \theta s C a}{2 \alpha(2+s)}-\frac{(1-\alpha)(1-\theta) s C b}{2 \alpha(2+s)}
$$

Haciendo manipulaciones y sacando factores comunes, se llega a:

$$
q 2(C b)=\frac{a}{2+s}+\frac{c s}{2 \alpha(2+\alpha)}-\frac{C b}{2}\left(1+\frac{(1-\alpha) s}{\alpha(2+s)}\right)+\frac{(1-\alpha) \theta}{2 \alpha(2+s)}(C b-C a)
$$

Simplificando se llega a la ecuación (12):

$$
q 2(C b)^{*}=a /(2+s)+[s / 2 \alpha(2+s)] c-[(2 \alpha+s) / 2 \alpha(2+s)] C b+[(1-\alpha) \theta s / 2 \alpha(2+s)](C b-C a)
$$

